\title{
Scrubbing Wipes to Dislodge Dental Biofilm Can Remove and Inactivate Viruses from Tooth Surface: Perspectives for Covid-19 Prevention
}

\author{
D’Accolti Maria ${ }^{1}$, Roncati Marisa ${ }^{2}$, Soffritti Irene ${ }^{1}$, Siciliani Giuseppe ${ }^{3}$ and Caselli Elisabetta ${ }^{1 *}$ \\ ${ }^{1}$ Section of Microbiology, Department of Chemical and Pharmaceutical Sciences and LTTA, University of Ferrara, Ferrara, Italy \\ ${ }^{2}$ Master of Prosthesis and Implantology, Alma Mater Studiorum, Bologna University, Bologna, Italy \\ ${ }^{3}$ Dental School, Department of Translational Medicine, University of Ferrara, Ferrara, Italy
}

*Corresponding author: Elisabetta Caselli, Section of Microbiology, Department of Chemical and Pharmaceutical Sciences and LTTA, University of Ferrara, Ferrara, Italy, via Luigi Borsari 46 - 44121 Ferrara, Italy

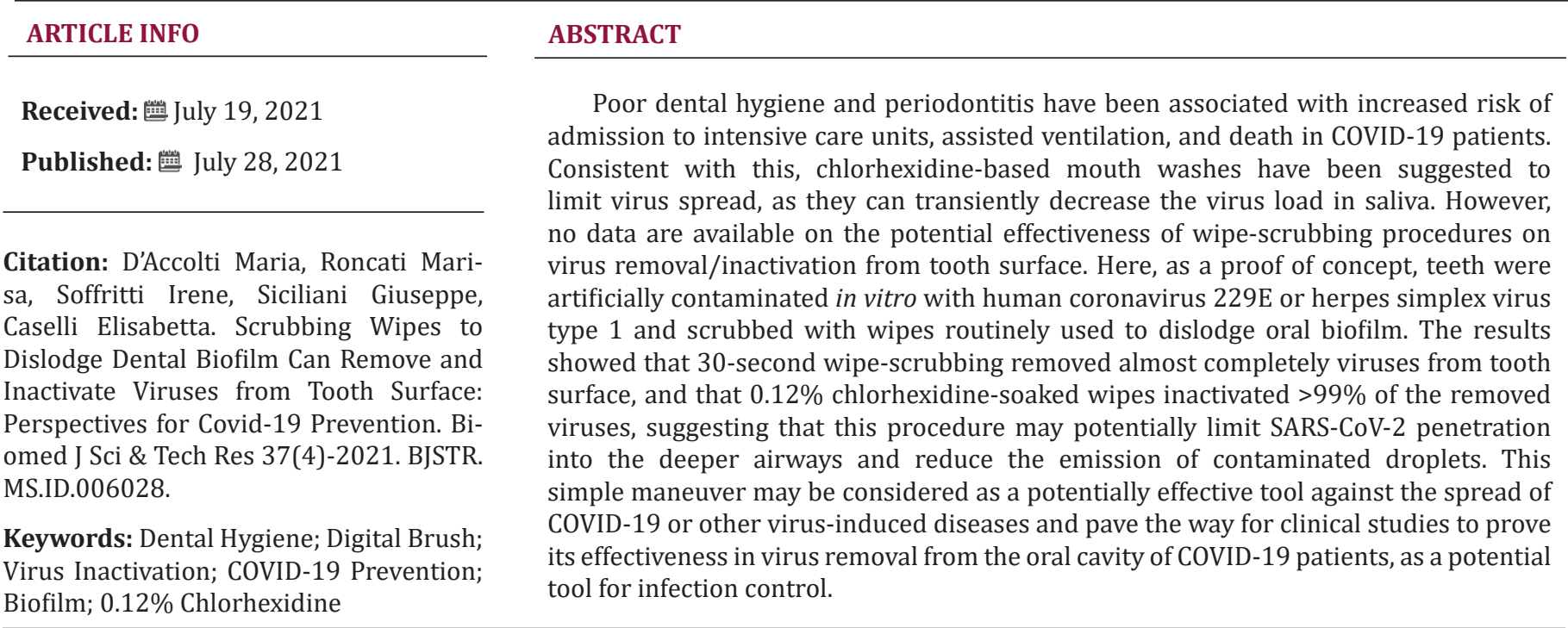

\section{Introduction}

Dysbiosis of the oral microbiome, often associated with periodontal inflammation, has been reported to impact systemic health and disease [1,2]. associating with worst progression and prognosis of many pathological conditions [2], including those originated by viral infections [3,4]. In particular, the oral microenvironment seems important for viruses entering the human body via the mouth, including SARS-CoV-2 and other respiratory and non-respiratory viruses [4-7]. Consistently, with regard to the current pandemics by SARS-CoV-2, the presence of gingival inflammation/periodontitis has been associated with a 3.5-fold increased risk admission to intensive care units (ICU), a 4.5 -fold greater risk of assisted ventilation, and a consistent impressive 8.81-fold higher risk of death in COVID-19 patients, independently from other concomitant risk factors [8].

Hence, neglected oral hygiene might act as a favoring cofactor for SARS-CoV-2, increasing the likelihood of severe COVID-19, and facilitating effective virus replication and penetration in the deep respiratory tree and worse course of the disease [9-11]. Based on these observations, it appears that proper oral hygiene may be instead important to control infection, limiting the establishment of virus infection and disease progression. In addition, dental hygiene procedures may be also provide direct inactivation of the virus, 
and oral antiseptics have been recently reported to be effective at inactivating human coronaviruses $(\mathrm{hCoV})$ in vitro $[12,13]$ and transiently decrease the SARS-CoV-2 load in saliva of two treated patients [14], suggesting that proper oral hygiene may limit virus transmission.

However, no data are available on the potential effectiveness of wipe-scrubbing procedures on virus removal/inactivation from tooth surface. Saline- or antiseptic-soaked wipes can be routinely used to dislodge dental biofilm, have a low-cost, and could be easily used multiple times during the day as a stand-alone procedure. Thus, they may represent an easy maneuver, repeatable all the needed times. As a proof of concept, here we aimed to assess in vitro the potential effectiveness of such dental hygiene maneuver against virus persistence on the tooth surface. To this purpose, natural extracted or prosthetic teeth were artificially contaminated with human coronavirus (as a surrogate of SARS-CoV-2) or herpes simplex virus 1 (as an example of viruses frequently detected in the mouth) were used to test the removal/inactivation ability of dental wipes.

\section{Materials and Methods \\ Viruses and Cells}

All the assays were performed using virus strains obtained from the international standard American Type Culture Collection (ATCC): the human coronavirus 229E (hCoV-229E, ATCC VR-740) and the human herpes simplex virus type 1 (HSV-1, ATCC VR-733). MRC- 5 cell line (human foetal lung fibroblast cells, ATCC CCL-171) and Vero cell line (monkey kidney fibroblast cells, ATCC CRL-1586) were used for hCoV-229E and HSV-1 propagation and titration, respectively. Both cell lines were cultured at $37^{\circ} \mathrm{C}+5 \% \mathrm{CO}_{2}$ in Minimal Essential Medium Eagle (EMEM) medium supplemented with $10 \%$ foetal bovine serum (FBS), 2mM L-Glutamine, 100U/ $\mathrm{mL}$ penicillin, and $100 \mu \mathrm{g} / \mathrm{mL}$ streptomycin (cell culture complete medium) (Gibco, Grand Island, NY).

\section{Teeth Contamination Assay}

All the assays were performed in vitro on natural extracted or prosthetic teeth, sterilized by autoclave, under a Biohazard flow-chamber. Teeth were artificially contaminated with $50 \mu \mathrm{l}$ of phosphate-buffered solution (PBS) containing 107 TCID50 of hCoV229E or HSV-1, allowed to dry for 90 minutes, and then treated by 30-second scrubbing either with saline-soaked (Digital Brush Baby, Enacare, Micerium, Avegno, GE, Italy) or with $0.12 \%$ chlorhexidinesoaked wipes indicted for biofilm removal (Digital Brush, Enacare, Micerium, Avegno, GE, Italy). Negative and positive controls were also included in the assays, respectively represented by untreated teeth or $70 \%$ ethanol-treated teeth.

After treatment, wipes and teeth were separately placed in sterile tubes containing $1 \mathrm{ml}$ of cold EMEM medium supplemented with $2 \%$ FBS (titration medium) and vortexed for 30 seconds to detach residual virus from the tooth surface. Each sample was serially diluted in cold titration medium to quantify the residual virus load. Duplicate samples from three independent assays were analyzed for natural extracted and prosthetic teeth.

\section{Titration Assays}

The day before the assay, the appropriate target cells were seeded in 96-wells microplates at 1.5-2x104 density/well to obtain optimal confluence. Following the teeth contamination assay, the residual viruses recovered from the teeth surface were serially diluted in cold titration medium and seeded ( $0.1 \mathrm{ml} /$ well $)$ in 96-wells microplates containing sub-confluent monolayers of the appropriate target cell type to measure the residual virus load. Sestuplicate samples were evaluated for each dilution, following the Spearman-Karber method $[15,16]$. To visualize the virus-induced cytopathic effect (CPE), hCoV-229E and HSV-1 infected cells were respectively incubated at $35^{\circ} \mathrm{C}+5 \% \mathrm{CO}_{2}$ for 7 days, and at $37^{\circ} \mathrm{C}$ $+5 \% \mathrm{CO}_{2}$ for 2 days. The infectious titre was expressed as Tissue Culture Infectious Dose 50 (TCID50) per ml, using the SpearmanKarber method.

\section{Statistical Analyses}

The significance of the collected results was assessed by t-test. Data are presented as mean \pm standard deviation (SD) values. A p value $<0.05$ was considered significant.

\section{Results}

\section{In vitro Removal and Inactivation of Viruses from Teeth by Wipe Scrubbing}

As a proof of concept, wipe dental scrubbing was applied in vitro, on natural extracted or prosthetic teeth sterilized by autoclave and artificially contaminated with about 107 TCID50 of hCoV-229E or HSV-1. Viruses were allowed to dry for 90 minutes on tooth surface, then each contaminated tooth was treated by 30 -second gentle scrubbing either with commercially available saline-soaked or $0.12 \%$ chlorhexidine-soaked wipes indicated for dental biofilm removal (Figure 1). Negative and positive assay controls were respectively represented by lack of treatment and by treatment with a virucidal solution consisting of $70 \%$ ethanol.

Each wipe and tooth were then separately analyzed for virus content. Briefly, each sample was placed in sterile tubes containing $1 \mathrm{ml}$ of cold EMEM medium supplemented with 2\% FBS (titration medium), vortexed for 30 seconds to detach residual virus, and serially diluted (10-fold dilutions) in cold titration medium. The quantification of the residual virus load was measured in duplicate samples from three independent assays, for both natural and prosthetic teeth. The results showed a slight reduction of the original virus titre after 90 minutes of desiccation on tooth surface, indicating that this procedure was reliable for the subsequent assessment of the antiviral properties of dental wipes (Figure 2). 


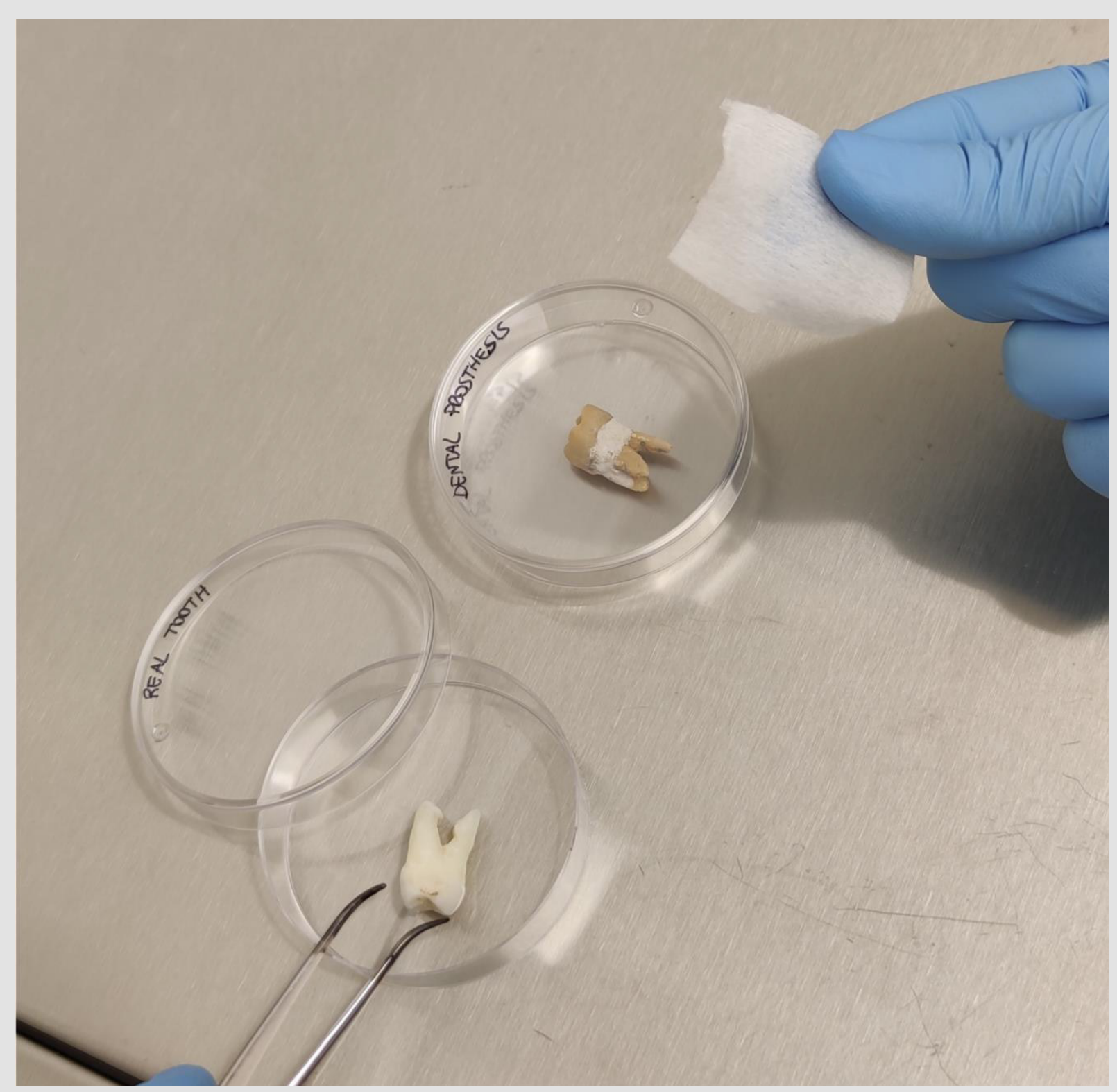

Figure 1: In vitro assays to evidence wipe-scrubbing ability to remove viruses from tooth surface. Natural or prosthetic teeth were artificially contaminated in their dental crown section with the human coronaviruses 229E or herpes simplex virus type 1, let too dry for 90 minutes, then scrubbed for 30 seconds with the indicated wipe.

Notably, following 30-second scrubbing with saline- or chlorhexidine-soaked wipes, virtually no residual hCoV-229E virus was detectable on tooth surface $(\mathrm{p}<0.001)$ (Figure 2A). HSV1 titre was decreased $99.99 \%$ (4 Logs) by saline-soaked wipes ( $\mathrm{p}<0.01$ ) and $99.999 \%$ (6 Logs) by chlorhexidine-soaked wipes
(Figure 2B). Interestingly, however, the viruses removed from tooth surface, entrapped in the wipes, retained their infectivity when using saline-soaked wipes, whereas were inactivated $>99 \%$ by $0.12 \%$ chlorhexidine-soaked wipes $(\mathrm{p}<0.001)$, No differences were observed between natural and prosthetic teeth. 


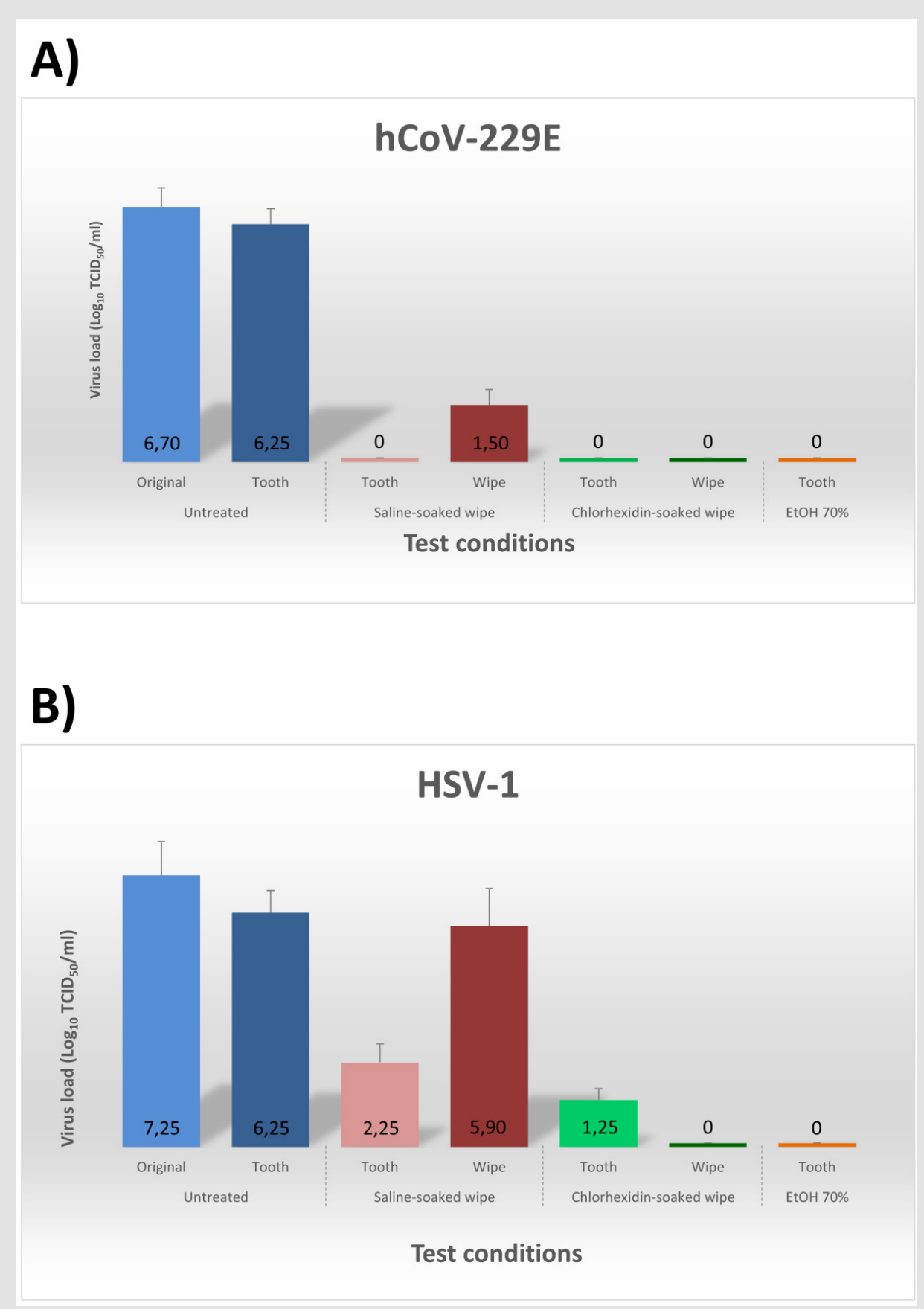

Figure 2: Virus removal by saline-soaked and chlorhexidine-soaked wipes used for oral biofilm removal. Extracted teeth or dental prostheses were artificially contaminated with the indicated viruses, allowed to dry for 90 minutes, and then untreated (negative control), or treated with the indicated wipes (saline- or $0.12 \%$ chlorhexidine-soaked), or with $70 \%$ ethanol $(\mathrm{EtOH}$, positive control). The virus load originally used to contaminate the teeth (Original), and that recovered from tooth surface (Tooth), and wipes (Wipe) are expressed as Log10 of Tissue Culture Infectious Dose 50 (TCID ${ }_{50}$ ) per ml. The results represent mean \pm SD values of duplicate samples (for both natural and prosthetic teeth) in three independent assays. A) Human coronavirus type 229E, hCoV-229E. B) Human herpesvirus 1, HSV-1.

\section{Discussion}

The data presented here show for the first time that dental scrubbing with wipes routinely used for dental biofilm dislodgment can effectively remove viruses from tooth surface in in vitro assays, providing a proof of concept for their use in clinical studies. The results were almost superimposable for both the enveloped viruses tested, chosen due to the close structural similarity of hCoV-229E with SARS-CoV-2, and to the high frequency of detection of HSV-1 in the oral cavity. These results may thus predict the effect against SARS-CoV-2 and confirm what was observed with chlorhexidine mouthwashes [14]. 
So far, many studies have emphasized the possible role of proper oral hygiene as a tool for controlling SARS-CoV-2 infection and transmission $[11,14,17,18]$, but no data are available on the effectiveness of simple wipe-scrubbing techniques in the removal and inactivation of viruses from tooth surface. The results reported here indicate that a brief and simple scrubbing technique may be very efficient in removing and neutralizing viruses eventually present on the tooth surface and that regular use of wipes for biofilm removal could potentially have a double effect: i) avoiding further penetration of the virus into the deeper airways in the infected subject, thus preventing the risk of a worse course of the disease, ii) reducing the emission of contaminated droplets, thus potentially helping to limit the risk of contagion.

These results highlight the potential of mechanical biofilm removal from teeth surface as a tool toward the SARS-CoV-2 infection control and pave the way for clinical studies to confirm the effect of such maneuvers in vivo in COVID-19 patients. Simple dental hygiene procedures may in fact contribute to prevent and control virus spread and infection and, due to their ease of use, low costs, and absence of contraindications, might be considered as an adjunctive and possibly highly effective tool in COVID-19 and other viral infections control strategies [19].

\section{Conclusion}

Since a simple 30-second wipe-scrubbing with $0.12 \%$ chlorhexidine-soaked wipes removed and inactivated $>99 \%$ of the tested viruses from teeth surface, this procedure may potentially limit the penetration of viruses entering the body via the oropharynx (such as SARS-CoV-2), with important clinical outcomes. Furthermore, by reducing the emission of contaminated droplets, this easy maneuver may be considered as a potentially effective tool against the spread of COVID-19 or other virus-induced diseases.

\section{Acknowledgements}

We thank Micerium SpA (Genova, Italy) for providing the Digital Brush, and the Parma-Benfenati dental office for providing extracted and prosthetic teeth.

\section{Conflict of Interest}

The authors declare that they have no conflict of interest.

\section{References}

1. Deo PN, Deshmukh R (2019) Oral microbiome: Unveiling the fundamentals. J Oral Maxillofac Pathol 23(1): 122-128.

2. Caselli E, Fabbri C, D’Accolti M, Soffritti I, Bassi C, et al. (2020) Defining the oral microbiome by whole-genome sequencing and resistome analysis: the complexity of the healthy picture. BMC Microbiol 20: 120.
3. Cagna DR, Donovan TE, McKee JR, Eichmiller F, Metz JE, et al. (2019) Annual review of selected scientific literature: A report of the Committee on Scientific Investigation of the American Academy of Restorative Dentistry. J Prosthet Dent 120(6): 816-878.

4. Baghbani T, Nikzad H, Azadbakht J, Izadpanah F, Haddad Kashani $\mathrm{H}$ (2020) Dual and mutual interaction between microbiota and viral infections: a possible treat for COVID-19. Microb Cell Fact 19(1): 217.

5. Wilks J, Beilinson H, Golovkina TV (2013) Dual role of commensal bacteria in viral infections. Immunol Rev 255(1): 222-229.

6. Wilks J, Golovkina T (2012) Influence of microbiota on viral infections. PLoS Pathog 8(5): e1002681.

7. Li N, Ma WT, Pang M, Fan QL, Hua JL (2019) The Commensal Microbiota and Viral Infection: A Comprehensive Review. Front Immunol 10: 1551.

8. Marouf N, Cai W, Said KN, Daas H, Diab H, et al. (2021) Association between periodontitis and severity of COVID-19 infection: A casecontrol study. J Clin Periodontol 48(4): 483-491.

9. Botros N, Iyer P, Ojcius DM (2020) Is there an association between oral health and severity of COVID-19 complications? Biomed J 43(4): 325327.

10. Herrera D, Serrano J, Roldan S, Sanz M (2020) Is the oral cavity relevant in SARS-CoV-2 pandemic? Clin Oral Investig 24(8): 2925-2930.

11. Sampson V, Kamona N, Sampson A (2020) Could there be a link between oral hygiene and the severity of SARS-CoV-2 infections? Br Dent J 228(12): 971-975.

12. Meyers C, Robison R, Milici J, Alam S, Quillen D, et al. (2021) Lowering the transmission and spread of human coronavirus. J Med Virol 93(3): 1605-1612.

13. Pelletier JS, Tessema B, Frank S, Westover JB, Brown SM, et al. (2021) Efficacy of Povidone-Iodine Nasal and Oral Antiseptic Preparations Against Severe Acute Respiratory Syndrome-Coronavirus 2 (SARSCoV-2). Ear Nose Throat J 100: 192S-196S.

14. Yoon JG, Yoon J, Song JY, Yoon SY, Lim CS, et al. (2020) Clinical Significance of a High SARS-CoV-2 Viral Load in the Saliva. J Korean Med Sci 35(20): e195.

15. Spearman C (1908) The Method Of 'Right and Wrong Cases' ('Constant Stimuli') Without Gauss's Formulae. British Journal of Psychology, 190419202 (3): 227-242.

16. Karber G (1931) Contribution to the collective treatment of series pharmacological experiments. (Beitrag zur kollektiven Behandlung pharmakologischer Reihenversuche). Archiv f experiment Pathol u Pharmakol 162: 480-483.

17. Mateos Moreno MV, Obrador AM, Marquez VA, Ferrer Garcia MD (2021) Oral Antiseptics against Coronavirus: In Vitro and Clinical Evidence. J Hosp Infect 113: 30-43.

18. Koch Heier J, Hoffmann H, Schindler M, Lussi A, Planz O (2021) Inactivation of SARS-CoV-2 through Treatment with the Mouth Rinsing Solutions ViruProX((R)) and BacterX((R)) Pro. Microorganisms 9(3): 521.

19. Roncati M (2020) A prevention recommendation, no contraindications. Pending a vaccine, protect against viruses and bacteria. In Sci J Res Dentistry 4(2): 33-34. 
ISSN: 2574-1241

DOI: 10.26717/BJSTR.2021.37.006028

Caselli Elisabetta. Biomed J Sci \& Tech Res

(C) (P) This work is licensed under Creative BY Commons Attribution 4.0 License

Submission Link: https://biomedres.us/submit-manuscript.php

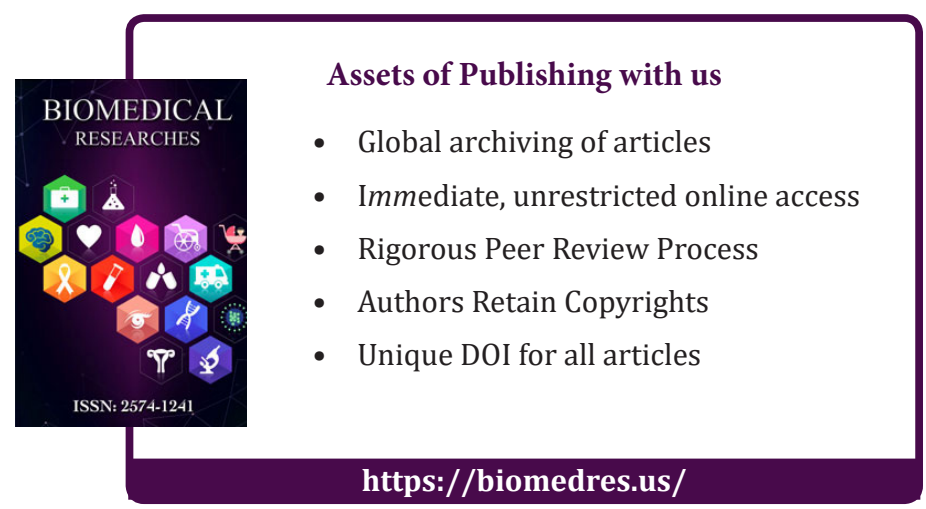

